\title{
On the Performance Analysis of the ICA Algorithms with Maternal and Fetal ECG signals Inputs and Contaminated Noises
}

\author{
S.D.Parmar \\ ECE Department \\ U.V.Patel College of Engineering \\ Ganpat Vidyanagar, Kherva. India
}

\author{
Bhuvan Unhelkar \\ School of Computing and Mathematics \\ University of Western Sydney, Australia
}

\begin{abstract}
This paper evaluates the performance of some major Independent Component Analysis (ICA) algorithms like Cardoso's Joint Approximate Diagonalization of Eigen matrices (JADE), Comon's algorithm and Optimized Generalized Weighted Estimator (OGWE) ICA algorithm in a biomedical blind source separation problem. Independent signals representing Fetal ECG (FECG) and Maternal ECG (MECG) are generated and then mixed linearly in the presence of white or pink noise to simulate a recording of electrocardiogram. While ICA has been used to extract FECG, very little literature is available on its performance in clinical environment. So there is a need to evaluate performance of these algorithms in Biomedical. To quantify the performance of ICA algorithms, two scenarios, i.e., (a) different amplitude ratios of simulated maternal and fetal ECG signals, (b) different values of additive white Gaussian noise or pink noise, were investigated. Higher order and second order performances were measured by performance index and signal-to-error ratio respectively. The selected ICA algorithms separate the white and pink noises equally well. This paper reports on the performance of the ICA algorithms.
\end{abstract}

\section{Categories and Subject Descriptors}

I.1.2 [Algorithms]: Analysis of Algorithm, I.5.4 ApplicationsSignal Processing

\section{General Terms}

Algorithm, Performance, Experimentation.

\section{Keywords}

BSS, ICA, Biomedical Signal Processing.

\section{INTRODUCTION}

The Electro Cardiogram (ECG) of an adult describes the electrical activity of the heart. This ECG is an important tool for the physician for identifying abnormalities in the heart activity. Similarly, it is important to obtain the Fetal Electro Cardiogram (FECG) of an unborn baby to trace possible problems in its heart activity. Most methods for acquiring the FECG are invasive which require placing an electrode on the fetal scalp. This procedure is available during delivery time only. It is important to try and find non-invasive techniques for earlier diagnosis. Obtaining FECG from recordings of electrodes on the mother's skin is fundamentally equivalent to the adult ECG with additional difficulties. The FECG is generated from a very small heart so the signal amplitude is low. Noise from electromyograpic activity affects the signal due to its low voltage. Another interesting source is the maternal ECG (MECG), which can be 5-1000 times higher in its intensity. There is no place to put an electrode on the mother's skin and to receive just the fetal signal without the signal being addled by the mother's signal. In all cases where the FECG is observed, the MECG is higher in magnitude. Hence eliminating the MECG from the recorded signal is very important [1] for good fetal reading.

Technically, the above problem can be thought of as a set of desired and undesired signals linearly mixed to produce another set of body surface signals. These signals are assumed to be non-Gaussian (except the random noise signal) and independent. ICA decomposes the mixed signals into as many statistically independent components as possible. ICA has been used to extract FECG [2][3][4][5]. However, very little literature is available on its performance in clinical environment. This needs an evaluation of its performance in clinical environment. Several ICA algorithms have been proposed. In paper [6], we have evaluated the performance of Optimized Generalized Weighted Estimator (OGWE) ICA algorithm. In this paper, we have evaluated the performance comparisons of Cardoso's joint approximate diagonalization of Eigen matrices (JADE) algorithm [7], Comon's algorithm [9], and OGWE algorithm [11] in a biomedical blind source separation problem. The signals, which are best suited for ICA, are designed to be biologically motivated for independent FECG and MECG. They are linearly mixed. The ICA separation produces independent FECG and MECG estimates.

\section{METHODOLOGY}

We consider the classical ICA model with instantaneous Mixing

$$
\mathbf{x}=\text { As }+\mathbf{n}
$$

where the sources $s=\left[s_{1}, s_{2} \ldots s_{n}\right]^{T}$ are mutually independent random variables and $A_{n \times n}$ is an unknown invertible mixing matrix and noise $n=\left[n_{1}, n_{2}, \ldots, n_{n}\right]^{T}$. The goal is to find only from observations, $\mathbf{x}$, a matrix $\mathrm{W}$ such that the output

$$
\mathbf{y}=\mathrm{Wx}
$$

is an estimate of the possible scaled and permutated source vector $\mathbf{s}$.

Preprocessing for ICA:

Some preprocessing is useful before attempting to estimate $\mathrm{W}$.

i. The observed signals should be centered by subtracting their mean value $\mathrm{E}\{\mathrm{x}\}$

$$
\mathbf{x}=\mathrm{x}-\mathrm{E}\{\mathrm{x}\}
$$

ii. Then they are whitened, which means they are linearly transformed so that the components are uncorrelated and has unit variance. 
iii. Whitening can be performed via eigenvalue decomposition of the covariance matrix, $\mathbf{V} \mathbf{\Lambda} \mathbf{V}^{\mathrm{T}}, \mathrm{V}$ is the matrix of orthogonal eigenvectors and $\boldsymbol{\Lambda}$ is a diagonal matrix with the corresponding eigenvalues. The whitening is done by multiplication with the transformation matrix $\mathbf{P}$

$$
\begin{aligned}
& \mathbf{P}=\mathbf{V} \boldsymbol{\Lambda}^{1 / 2} \mathbf{V}^{\mathrm{T}} \\
& \mathbf{Z}=\mathbf{P} \mathbf{x}
\end{aligned}
$$

This is closely related to principal component analysis. The covariance of the whitened data $\mathrm{E}\left\{\mathbf{Z} \mathbf{Z}_{\mathrm{T}}\right\}$ equals the identity matrix. For simplicity, let $\mathbf{x}$ be the centered mixed vector $x^{\wedge}$, i.e. $\mathbf{x}=\mathrm{x}^{\wedge}$

Jutten and Herault provided one of the first significant approaches to the problem of blind separation of instantaneous linear mixtures [12]. Since then, many different approaches have been attempted by numerous researches using neural networks, artificial learning, higher order statistics, minimization of mutual information, beam forming and adaptive noise cancellation, each claiming various degrees of success. Several algorithms exist for blind source separation. We evaluate the performance of some major ICA algorithms in a blind source separation problem. This section presents a brief description of the respective approaches of the algorithm.

\subsection{JADE Algorithm}

The JADE algorithm [7] relies on second and fourthorder cumulants to separate the sources. SOS is used to obtain a whitening matrix $\mathbf{Z}$ from the sample covariances. To reduce the computational load, only the $\boldsymbol{n}$ most significant eigen pairs of fourth order cumulants obtained from the whitened process are joint diagonalized by unitary matrix $\mathbf{U}$. The separated matrix can be estimated as $\mathbf{U}^{\dagger} \mathbf{Z}$, where $\dagger$ represents pseudo inverse. The JADE contrast function [8] is the sum of squared fourth order cross cumulants

$$
\phi^{J A D E} Y=\sum_{i j k l \neq i i k l}\left(\mathbf{Q}_{\mathrm{ijlk}}\right)^{2}
$$

As this algorithm uses cross-cumulants, there is no need to go for gradient descent and hence there is no chance of divergence. In addition, there is no problem of updating the weights and tuning the parameters.

\subsection{Comon's Algorithm}

A specific contrast function is proposed in[9], based on minimization of mutual information between the components at the output of separator (which is directly related to KullbackLeibler divergence between the output vector probability density function (pdf) and it's pdf if it was made of independent components). After some manipulations on the Edgeworth expansion [10] of the source joint pdf, the contrast function simplifies into sum of the output squared $r$ th-order marginal cumulants:

$$
\Psi_{\mathrm{r}} \quad \hat{\mathbf{Q}}=\sum_{\mathrm{i}=1}^{\mathrm{q}}\left(\mathrm{k}^{\mathrm{s}} \underset{i i \ldots i}{\mathrm{~s} \ldots i}\right)^{2}
$$

Note that the criterion depends on $\hat{\mathbf{Q}}$ through $\mathbf{S}=\hat{\mathbf{Q}}^{-1} \mathbf{z}$.

At 4th-order, the above contrast becomes the output-squared kurtosis:

$$
\Psi_{4} \quad \hat{\mathbf{Q}}=\sum_{\mathrm{i}=1}^{\mathrm{q}}\left(\mathbf{k}_{\underline{\text { iiii }}}^{\mathrm{s}}\right)^{2}
$$

\subsection{OGWE Algorithm}

In OGWE (Optimized Generalized Weighted Estimator) [11], the marginal entropy contrast function $\left(\Phi^{\mathrm{ME}}\right)$ is written in terms of second-order and fourth-order cumulants, and then it is minimized for all possible distributions for the sources $\mathbf{s}$, it follows that

$\Phi^{\mathrm{ME}}(\mathbf{y}) \approx 1 / 48 \Phi_{24}^{\mathrm{ME}}(\mathbf{y})=-1 / 48 \sum_{\mathrm{i}}\left(\mathbf{C}_{\text {iiii }}{ }^{\mathrm{y}}\right)^{2}$

where, for zero-mean signals, $\boldsymbol{C}_{i i i i}{ }^{y}=E\left\{y_{i}{ }^{4}\right\}-3 E\left\{y_{i}{ }^{2}\right\}$ are the marginal cumulants or autocumulants. The whitening matrix $\mathrm{P}$ is computed to whiten the vector $\mathbf{x}$ and the vector $\mathbf{y}=\mathrm{Px}$ is formed. For $1 \leq \mathrm{p} \leq \mathrm{q} \leq \mathrm{n}$ one sweep, the given angle $\theta_{p q}=\theta_{G W E}$ is computed [11]. If $\theta_{p q}>\theta_{\min }$, the pair $\left(Z_{p}, Z_{q}\right)$ is rotated by $\theta_{\mathrm{pq}}$ according to Eq.(9) and also the rotation matrix $R$ is updated until number of iterations satisfies. Then the demixing matrix $\mathrm{W}$ $=\mathrm{RP}$ and the independent sources are estimated as $\hat{\mathbf{s}}=\mathrm{W} \mathbf{x}$.

\section{EXPERIMENT SETUP}

As the different noise in the biomedical system grows, it becomes necessary to improve the efficiency of common biomedical resources. Independent Component Analysis (ICA) use as an advanced tool for blind suppression of interfering FECG and MECG signals contaminated by noise. The role of ICA is to provide a mitigated FECG signal to the conventional analysis. As the different noise in the biomedical system grows, also the ratio of FECG and MECG varies in amplitude; it becomes necessary to measure the performance of ICA algorithm.

\subsection{Signal generation}

The observed signals (Fig 1) at the electrodes were simulated by taking two different ECG signals from the MIT-BIH (Massachusetts Institute of Technology-Beth Israel Hospital Arrhythmia Laboratory) database [13]. These signals are sampled at $360 \mathrm{HZ}$. The FECG is generated from a very small heart so the signal amplitude is low. Noise from electromyograpic activity affects the signal due to its low voltage. Another interesting source is the maternal ECG (MECG), which can be 5-1000 times higher in its intensity. To simulate real conditions, the second signal (assumed as FECG) was 10 or 1000 times less in amplitude and with double the number of cycles/second compared to the first (assumed as MECG). We remove the mean of the original ECG signals and normalize the two ECG signals to unity. As a result, the desired maternal to fetal amplitude ratio can be obtained by multiplying the signal with that constant.
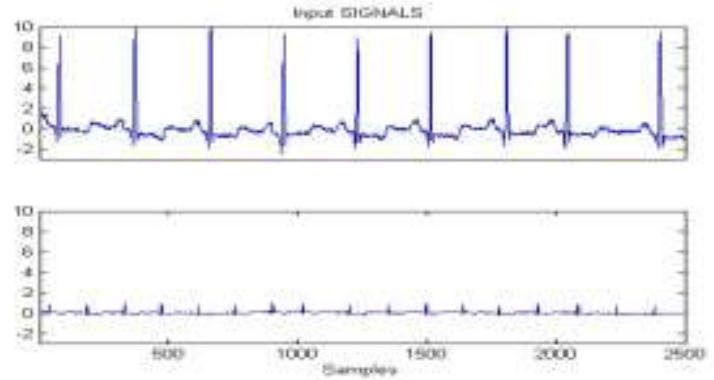

Figure 1 Generation of MECG and FECG signals 


\subsection{Linear mixing and Noise}

We set the mixing coefficient between the MECG and FECG signals to

$$
A=\left(\begin{array}{cc}
-0.1430 & -2.2008 \\
0.9943 & -0.8061
\end{array}\right)
$$

The additive white Gaussian noise was generated by MATLAB. In the body many electrical signals are time correlated and would be modeled better by colored noise instead of white noise [14]. The pink noise was created with a Fourier domain generator with the power spectrum given by

$$
P_{m}(f) a f^{-\beta} \beta>0
$$

These noise records (consider as $\mathrm{n}$ in Equation 1) were added to the mixed signals with a specified signal to noise ratio (SNR) (which is measured with respect to the mixed signals in the given channel). In this way, the SNR in both channels are the same, but the amplitudes are quite different. The results in our two channels are the simulated FECG and simulated MECG.

\subsection{Performance evaluation}

To quantify the higher order performance of the demixing we use the performance index, PI. This is a measure on the global system matrix $\mathrm{P}=\mathrm{WA}$ suitable for the degeneracy conditions $\mathrm{W}=$ $\mathrm{A}^{-1}$ and is calculated as

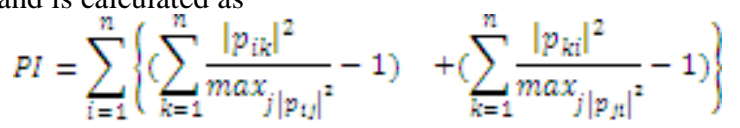

where $p_{i j}$ is the $(i, j)$ th element of the global system matrix $P=$ WA and $\max _{j} \mathrm{p}_{\mathrm{ij}}$ represents the maximum value among the elements in the ith row vector of $P, \max _{j} p_{j i}$ does the maximum value among the elements in the ith column vector of $\mathrm{P}$. When perfect signal separation is carried out, the performance index $P I$ is zero. Since we are looking at the estimation of the FECG and MECG signals, we also consider the $S E R$, which is a second order measure. The SER was obtained by using the following relation

$$
S E R=10 \log _{10}\left(\frac{E|s(t)|^{2}}{E|e(t)|^{2}}\right)
$$

where $\mathrm{s}(\mathrm{t})$ is the desired signal and $\mathrm{e}(\mathrm{t})=\mathrm{s}(\mathrm{t})-\mathrm{s}(\mathrm{t})$ is the error. Here $\hat{s}(\mathrm{t})$ is the estimated source signal and $\hat{s}(\mathrm{t})$ and $\mathrm{s}(\mathrm{t})$ should be at the same energy level and phase while calculating $\mathrm{e}(\mathrm{t})$.

\section{EXPERIMENTAL RESULTS AND DISCUSSION}

The ICA algorithms separations of our simulation are shown in Fig 2, 3, 4, 5, 6 and 7 for comparisons. Fig. 2 show extracted output SNR of MECG for 10, 50, 100, 500 and 1000 amplitude ratios of simulated MECG and FECG signals in presence of Gaussian noise. As MECG to FECG ratio varies from 10 to 100 the output SNR of MECG increases. Results show that there is no changes in output SNR for 0 to $20 \mathrm{~dB}$ input SNR, but gives different output SNR for 20 to $30 \mathrm{~dB}$ input SNR. To make more robust, when the ratio varies from 100 to 1000, in this duration JADE and OGWE perform well, this gives more output SNR compare to Comon's algorithm. The output SNR results for MECG are shown for pink noise in Fig 3. MECG to FECG ratio varies from 10 to 1000 the output SNR of MECG increases. For pink and for 0 to $25 \mathrm{~dB}$ input SNR all three algorithms performance is easily differentiated. Again JADE and OGWE perform well.

The output SNR results for FECG from the ICA algorithms separation of our simulation are shown for Gaussian noise in Fig 4 and for pink noise in Fig 5. All the algorithms are able to extract FECG considerably if the amount of input SNR is of the order of $10 \mathrm{~dB}$ or less. Even if the SNR approach $0 \mathrm{~dB}$ all the algorithms are still able to extract the R wave. As MECG to FECG ratio varies from 10 to 1000 the output SNR of FECG decreases. For ratio from 100 to 1000 , results show that there are changes in output SNR for 0 to $10 \mathrm{~dB}$ input SNR. Comon's algorithm performances good in this duration.

All algorithms separate the white and pink noise equally well. By processing the data we clearly achieve a better second order estimate of the FECG independent of the noise color. In fact, the SNR of the extracted FECG is equivalent to the SNR specified for the added noise up to $30 \mathrm{~dB}$ level. In this duration, JADE and OGWE algorithm performs well.

The Performance Index results from the ICA algorithms separation of our simulation are shown for Gaussian noise in Fig 6 and for pink noise in Fig 7. The output performance index results show a peak at in between 0 to $8 \mathrm{~dB}$ input $\mathrm{SNR}$, but as the MECG to FECG ratio increase from 10 to 1000 this peak shift to other input SNRs. Finally peak decrease for more MECG to FECG ratio and performance index becomes lower. The performance index of the performance matrix $\mathrm{P}=\mathrm{WA}$ indicates the same decay in higher order separation. As the noise contamination becomes dominant, the demixing performance is poorer between 0 to $5 \mathrm{~dB}$. Performance index zero indicates a good separation of the two signals. The performance of the Comon's algorithm is slightly less compared to the other two algorithms. JADE algorithm has the least computational cost and also no parameters to tune.

\section{CONCLUSIONS}

In this paper we have calculated the performance of the JADE, OGWE and Comon's ICA algorithms in a simple electro physiologically motivated Blind Source Separation (BSS) problem to extract the FECG. Using simulated independent signals from the pregnant woman skin (FECG and MECG), we observe that the BSS performance of the algorithms are unaffected by noise as long as the added noise does not exceed the corruption due to mixing. By processing the data we clearly achieve a better estimate of the FECG independent of the noise color. We also observe that amplitude ratios of simulated MECG and FECG signal increase affects the BSS performances of the algorithms. As the amplitude ratios of simulated MECG and FECG signal increase, all algorithms are able to extract the sources but with different performance index. Even for high value of performance index, quality of separated signals is quite satisfactory for all algorithms. The performance of the JADE and OGWE algorithm are slightly better compared to the Comon's algorithm. 


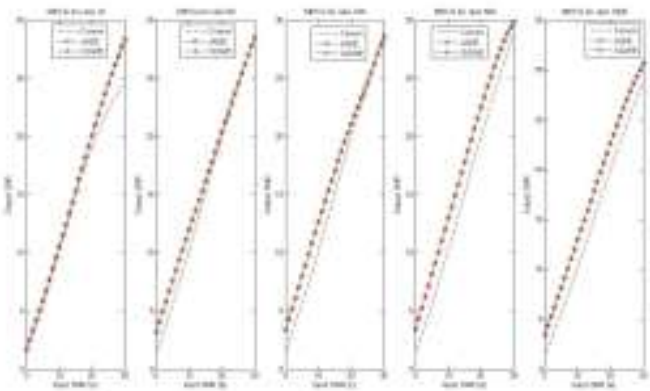

Figure 2 show extracted output SNR of MECG for (a) 10 (b) 50 (c) 100 (d) 500 and (e) 1000 amplitude ratios of simulated MECG and FECG signals in presence of Gaussian noise.

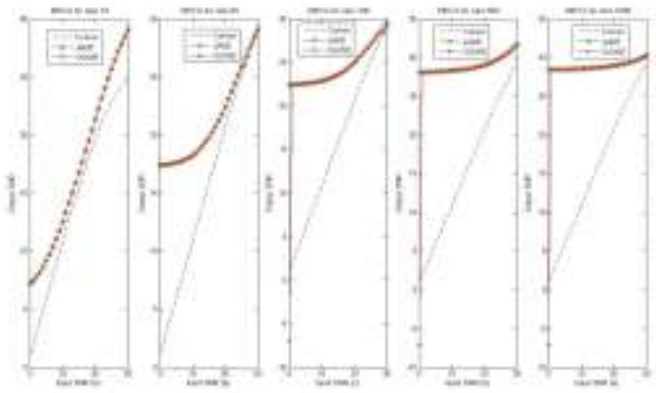

Figure 3 show extracted output SNR of MECG for (a) 10 (b) 50 (c) 100 (d) 500 and (e) 1000 amplitude ratios of simulated MECG and FECG signals in presence of Pink noise.

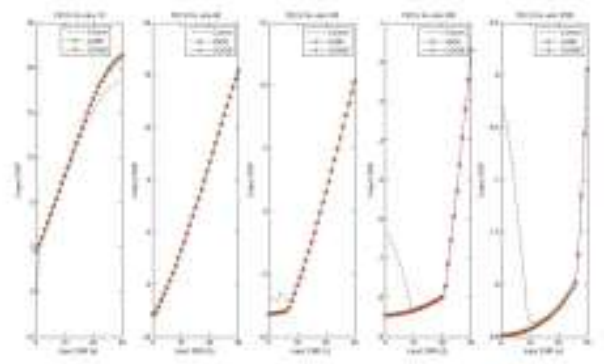

Figure 4 show extracted output SNR of FECG for (a) 10 (b) 50 (c) 100 (d) 500 and (e) 1000 amplitude ratios of simulated MECG and FECG signals in presence of Gaussian noise.

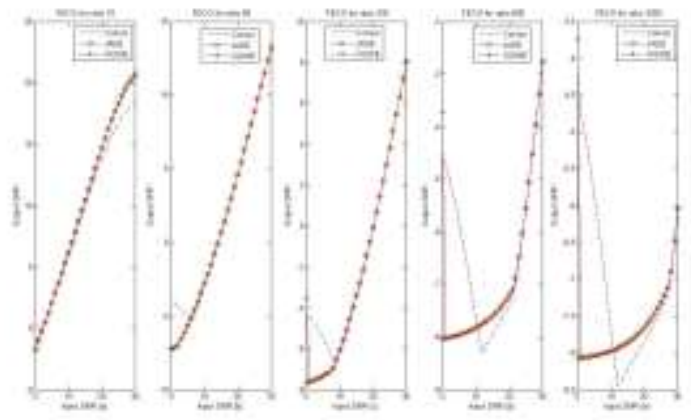

Figure 5 show extracted output SNR of FECG for (a) 10 (b) 50 (c) 100 (d) 500 and (e) 1000 amplitude ratios of simulated MECG and FECG signals in presence of Pink noise.

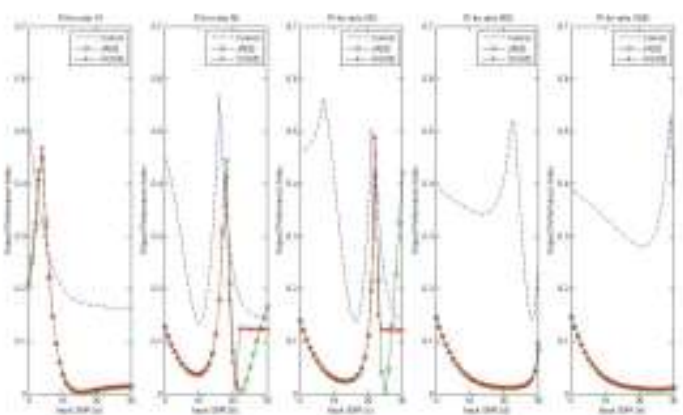

Figure 6 show output Performance Index for (a) 10 (b) 50 (c) 100 (d) 500 and (e) 1000 amplitude ratios of simulated MECG and FECG signals in presence of Gaussian noise.

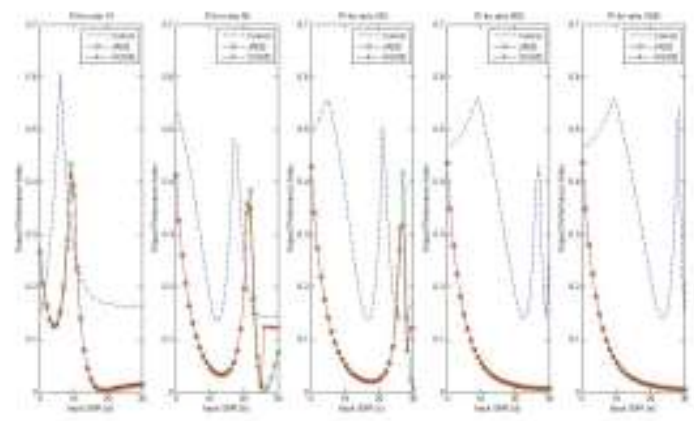

Figure 7 show output Performance Index for (a) 10 (b) 50 (c) 100 (d) 500 and (e) 1000 amplitude ratios of simulated MECG and FECG signals in presence of pink noise.

\section{REFERENCES}

[1] Amit Kam and Arnon Cohen, "Maternal ECG ellimination and Foetal ECG detection-Comparision of several Algorithms," Procee. of the 20th annual international conference of the IEEE Engineeing in Medicine and Biology Society., vol. 20, No.1, pp-174-177, 1998.

[2] V.Zarzoso and A.Nandi, "Noninvasive fetal ECG extraction: Blind separation versus adaptive noise cancellation," IEEE trans, Biomed Engg., vol 48, No1, pp. 12-18, 2001.

Seungjin choi, A.Chichocki, S.Amari, "flexible independent component analysis", journal of VLSI Signal Processing, kluwer academic publishers., boston, 2000.

[3] S.D.Parmar and J.S.Sahambi, "A Comparative Survey on removal of MECG artifacts from FECG using ICA algorithms," Proceeding of International Conference on Intelligent Sensing and Imformation Processing-2004, Chennai-India, ICISIP-2004, pp-88-91.

[4] Seungjin choi, A.Chichocki, S.Amari, "Flexible independent component analysis", journal of VLSI Signal Processing, kluwer academic publishers. boston, 2000.

[5] S.D.Parmar and Bhuvan Unhelkar, "Separation Performance of ICA Algorithms in FECG and MECG signals contaminated by Noise", International Conference on Computing, Communication and Networking (ICCCN-2008), $18^{\text {th }}-20^{\text {nd }}$ December 2008, Karur-TN, India.

[6] S.D.Parmar and Bhuvan Unhelkar, "Performance of Optimized Generalized Weighted Estimator ICA algorithm 
on Biomedical Signals Contaminated by Noise", International Journal of Computer Applications Vol: 1 Issue: 2 pp. 29-33, 2010.

[7] J.F. Cardoso and A. Souloumiac, "Blind beamforming for non-Gaussian signals," IEE Proceeding- $F$., vol. 140 , no. 6, pp. 362-370, Dec-1993.

[8] J.F. Cardoso, "Higher order contrasts for independent component analysis," Neural computation, vol. 11, no. 1, pp. 157-192, Jan. 1997.

[9] P. Comon, "Independent component analysis, a new concept?," Signal processing (Special Issue Higher Order Statistics), vol. 36, no. 3, pp. 287-314, April 1994.

[10] P. Comon, "Contrasts for Multichannel Blind Deconvolution," IEEE Signal Processing Lett., vol. 3, pp.209-211, July 1996.
[11] Juan J. Murillo-Fuentes and Rafael Boloix-Tortosa, Francisco J. Gonzt'alez-Serrano, "Initialized Jacobi Optimization in Independent Component Analysis". 4th International Symposium on Independent Component Analysis and Blind Signal Separation (ICA2003), Nara, Japan, pp 1053-1058, April 2003.

[12] C.Jutten and J.Herault,"Blind separation of sources part I: An adaptive algorithm based on neuromimatic architecture," Signal Processing., vol.24, pp. 1-10, July 1991.

[13] MIT-BIH Database Distribution. [Online]. "Available: http://ecg.mit.edu".

[14] M.Potter, N.Gadhok, and W.Kinsner, "Separation performance of ICA on simulated EEG and ECG signals contaminated by noise," Proc. of the 2002 IEEE canadian conf. on Electrical and computing engg., pp.1099-1104, 2002. 